\title{
Analysis on Multistable Motion Characteristics of Supercavitating Vehicle
}

\author{
Tianhong Xiong, Yipin Lv, and Wenjun Yi \\ National Key Laboratory of Transient Physics, Nanjing University of Science and Technology, Nanjing 210094, China \\ Correspondence should be addressed to Tianhong Xiong; xiongtianhong@njust.edu.cn
}

Received 6 July 2017; Revised 22 September 2017; Accepted 10 October 2017; Published 6 November 2017

Academic Editor: Matteo Aureli

Copyright (C) 2017 Tianhong Xiong et al. This is an open access article distributed under the Creative Commons Attribution License, which permits unrestricted use, distribution, and reproduction in any medium, provided the original work is properly cited.

\begin{abstract}
Due to complex underwater environment, when the initial condition of launching is subjected to low external disturbance, the motion trace of a supercavitating vehicle might display many different motion states during underwater navigation. With the aim of addressing this problem, based on the dynamic map, in the present work the multistable phenomena of attractor coexistence of the supercavitating vehicle system under various initial conditions were analyzed and the initial condition effects on the multistable motion characteristics were investigated through the domains of attraction, time, and frequency. The results demonstrated that, unlike the ordinary dynamic systems, a supercavitating vehicle demonstrates multistable phenomena, such as the coexistence of the stable equilibrium point and the limit cycle and the coexistence of the limit cycle and the chaotic attractor, along with the coexistence of diversified limit cycles; under fixed system parameters, as the initial condition of launching varied, the vehicle displayed various motion states; in engineering practices, the initial condition of launching could be adjusted according to the domain of attraction, in order for the vehicle motion stability to be enhanced.
\end{abstract}

\section{Introduction}

Owing to the broad application prospect in underwater drag reduction, the supercavitation has attracted significant attention from scholars all over the world [1-3], whereas a high number of numerical simulations and experiments have been conducted to study the mechanisms and laws of formation, the development, and the stabilization of the supercavitation [4-6]. Nevertheless, a vehicle in supercavitation state is almost totally surrounded by a cavity; only the cavitator and the tail of the vehicle contact with water. A complex nonlinear force is generated when the tail of the vehicle collides with the cavity wall, which will cause vibrations and shocks of the vehicle. At present, the investigations of supercavitating vehicle both domestically and abroad mainly focus on the modeling and control of supercavitating vehicle dynamics [711]. Based on the hydrodynamics of supercavitating vehicle, Dzielski and Kurdila [7] established a four-dimensional nonlinear dynamic model and designed a controller by the accurate linear approach; Lin et al. [8] analyzed various bifurcation behaviors of the supercavitating vehicle based on the model established by Dzielski and Kurdila [7] and employed the washout-filter for the bifurcation control of the supercavitating vehicle. Nguyen and Balachandran [9] analyzed the nonlinear dynamic behaviors of the supercavitating vehicle based on the noncylindrical and nonsymmetric cavity and discussed the effectiveness of feedback control strategies with fin and cavitator inputs on the vehicle stability. Based on the supercavitation model proposed by Dzielski and Kurdila, Han et al. [10] described the supercavitation system model as feedback connection forms, composed of linear links and nonlinear links, according to the absolutely stable condition of the vehicle. Also, the supercavitating vehicle controller was designed with the state feedback pole assignment. Bai et al. [11] obtained the cavitation numbers and the cavity pattern data in motion of the vehicle by water tunnel experiments, corrected the supercavity model [7], designed the supercavitating vehicle controller with the LPV, and validated the control effect through the simulation approach.

In a nonlinear dynamic system, when the system parameters vary in certain ranges, multiple attractors often coexist in a certain region, known as the multistable phenomena [12]. The coexisting attractor and the initial conditions that lead to the generation of this attractor comprise the attraction 
domain of the attractor [13]. If an attractor's domain of attraction does not intersect with any unstable equilibrium, it is called a hidden attractor; otherwise it is called a selfexcited attractor [14-16]. As a complex nonlinear physical phenomenon, the multistability has been extensively studied in the fields of the PLL circuit model and the electronic machine model as well as the aircraft, whereas a high number of achievements have been acquired [17-20]. During underwater movement, due to the complex underwater environment, the supercavitating vehicle is easily affected by external disturbances; moreover, a supercavitating vehicle is a strongly nonlinear system, where the final motion state is, thereby, determined by the domain of attraction where the initial condition is located. When the initial condition is located near the boundary of the attraction domain, low disturbances or changes in the initial condition might lead to the multistable phenomenon of the coexisting attractor that a hidden attractor coexists with a self-excited attractor [13], along with completely different dynamic characteristics in the phase portrait, the time domain, and the frequency domain. The multistable motion characteristics of the supercavitating vehicle are the important basis for the supercavitating vehicle controller design but have been poorly investigated to date, which require in-depth analysis and discussions. Based on the dynamic model of the supercavitating vehicle, in the present work the multistable phenomena induced by various initial conditions of the launching supercavitating vehicle were analyzed, whereas the multistable motion characteristics of the supercavitating vehicle through the domain of attraction, the time domain, and the frequency domain were investigated.

\section{Dynamic Model of Supercavitating Vehicle}

The supercavitating vehicle model as described in [7] was adopted in the present work. A disk cavitator is located at the nose of the vehicle of $R_{n}$ in radius; the length of the vehicle is $L$; the fore-end is a cone and the aft-end is a cylinder, whereas the length ratio of the former to the latter is $1: 2$; the distance between the center of gravity and the nose is $17 / 28 \mathrm{~L}$; the radius of the tail is $R$. The origin of the coordinate of the supercavitating vehicle is located at the center of the disk cavitator at the vehicle nose; the $X$-axis is along the symmetry axis, the advance speed $V$ is parallel to the vehicle axis, and the $Z$-axis is vertical to the $X$-axis straightly downwards.

Cavitator not only creates and sustains supercavitation but also controls the hydrodynamic forces around the nose as control surface. The lift component of the hydrodynamic force on cavitator is [7]

$$
F_{\text {cavitator }}=\frac{1}{2} \pi \rho R_{n}^{2} V^{2} C_{x} \alpha_{c},
$$

where $R_{n}$ is cavitator radius, $\alpha_{c}=w / V+\delta_{c}, \delta_{c}$ is cavitator deflection angle, and drag coefficient is $C_{x}=C_{x 0}(1+\sigma)$, being $C_{x 0}=0.82$.

The fin also needs control moment provided by certain hydrodynamic forces, and the hydrodynamic force on the fin is [7]

$$
F_{\text {fin }}=-n \frac{1}{2} \pi \rho R_{n}^{2} V^{2} C_{x} \alpha_{f},
$$

where $n$ denotes the effectiveness of the fin, $\alpha_{f}=(w+q L) / V+$ $\delta_{e}$, and $\delta_{e}$ is deflection angle of the fin.

When vehicle travels with supercavity encapsulation, due to change in the relative position of vehicle against cavity, the contact between the tail and the cavity wall induces complicated nonlinear planing force that generates vibration and shock on the body. Planing force is expressed as [8]

$$
F_{\text {planing }}=-V^{2}\left[1-\left(\frac{R_{c}-R}{h^{\prime} R+R_{c}-R}\right)^{2}\right]\left(\frac{1+h^{\prime}}{1+2 h^{\prime}}\right) \alpha .
$$

When part of the tail is immersed in water, [8] gives the immersion depth $h^{\prime}$ :

$$
h^{\prime}= \begin{cases}0 & \frac{\left(R_{c}-R\right)}{R}>\frac{L|w|}{R V} \\ \frac{L|w|}{R V}-\frac{R_{c}-R}{R} & \text { otherwise. }\end{cases}
$$

Moreover, [8] gives the angle-of-attack $\alpha$ when the tail of supercavitating vehicle is immersed in water:

$$
\alpha= \begin{cases}\frac{w}{V}-\frac{\dot{R}_{c}}{V} & \frac{w}{V}>0 \\ \frac{w+\dot{R}_{c}}{V} & \text { otherwise, }\end{cases}
$$

where $R_{c}$ and $\dot{R}_{c}$ denote the cavity radius and the contraction rate at the tail of vehicle, respectively.

The supercavitating vehicle motion in the vertical plane was investigated. As shown in Figure 1, the vehicle dynamic modeling utilized the four state variables $z, w, \theta$, and $q$ to describe the supercavitating vehicle dynamics, where $w$ denotes the transverse speed, $\theta$ denotes the pitch angle, $q$ denotes the pitch rate, and $z$ denotes the vertical position of vehicle. According to the supercavitating vehicle model as described in [7], with the assumption that the vertical speed $V$ remained constant during the supercavitating movement and the transverse forces were balanced, through the hydrodynamic forces acted on the vehicle, the derived dynamic model of the supercavitating vehicle is

$$
\begin{aligned}
\left(\begin{array}{c}
\dot{z} \\
\dot{w} \\
\dot{\theta} \\
\dot{q}
\end{array}\right)= & \left(\begin{array}{cccc}
0 & 1 & -V & 0 \\
0 & a_{22} & 0 & a_{24} \\
0 & 0 & 0 & 1 \\
0 & a_{42} & 0 & a_{44}
\end{array}\right)\left(\begin{array}{l}
z \\
w \\
\theta \\
q
\end{array}\right) \\
& +\left(\begin{array}{cc}
0 & 0 \\
b_{21} & b_{22} \\
0 & 0 \\
b_{41} & b_{42}
\end{array}\right)\left(\begin{array}{l}
\delta_{e} \\
\delta_{c}
\end{array}\right)+\left(\begin{array}{l}
0 \\
c_{2} \\
0 \\
0
\end{array}\right) \\
& +\left(\begin{array}{c}
0 \\
d_{2} \\
0 \\
d_{4}
\end{array}\right) F_{\text {planing. }}
\end{aligned}
$$




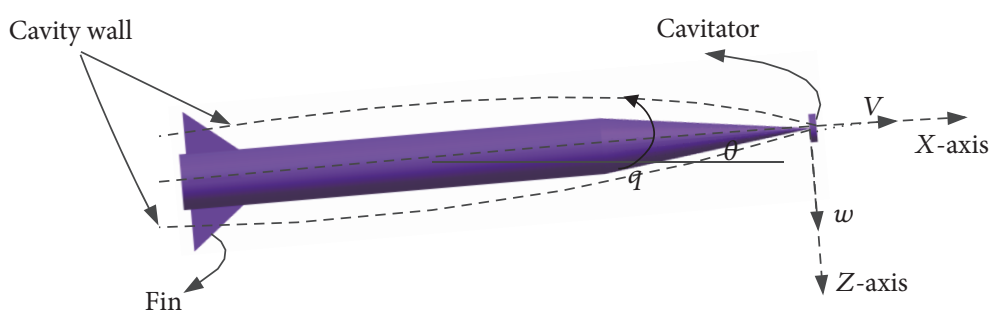

FIGURE 1: Schematic of supercavitating vehicle.

The details of the coefficients $a_{i j}, b_{i j}$, and $d_{i j}$ and other quantities used in (3)-(6) can be seen in [9].

The system parameters of the supercavitating vehicle were as follows [9]: $g=9.81 \mathrm{~m} / \mathrm{s}^{2}, m=2, R n=0.0191 \mathrm{~m}$, $R=0.0508 \mathrm{~m}, L=1.8 \mathrm{~m}, V \in[67.7,92.3] \mathrm{m} / \mathrm{s}, \sigma \in[0.0198$, $0.0368], n=0.5$, and $C_{x 0}=0.82$. Based on the system parameters assignment, Dzielski and Kurdila [7] proposed the classical control law: $\delta_{e}=0$ and $\delta_{c}=15 z-30 \theta-0.3 q$. In contrast, since the deflection angle of the fin $\delta_{e}$ was zero, the vehicle often lacked the supporting force provided by the fin to balance gravity, thereby leading to the vehicle instability. Therefore, in this work, the deflection angle of the cavitator $\delta_{c}=15 z-30 \theta-0.3 q$ remained unchanged, whereas the deflection angle of the fin $\delta_{e}$ was studied (given $\delta_{e}=$ $-k z$, where $k$ denotes the feedback control parameter for the vehicle pitch rate).

\section{Multistable Dynamic Characteristics of Supercavitating Vehicle}

3.1. Dynamic Map of Supercavitating Vehicle. As presented in Figure 2, under randomly selected initial conditions, the stable solution, the periodic solution, and the chaotic solution of the supercavitating vehicle dynamic model, as described in (6), were marked in blue, red, and green, respectively, in order for the dynamic map to be depicted with variable elements, including the cavitation number $\sigma$ and the feedback control gain of the deflection angle of fin $k$. It could be observed that the vehicle could move stably when the values were within the blue zone; the vehicle oscillated periodically when the values were within the red zone; the vehicle was in nonlinear oscillation when the values were within the green zone; the interface between the blue zone and the red zone was the critical transition line between the stable state and the periodic state; the interface between the red zone and the green zone denoted the transition between the periodic state and the chaotic state; the black zone denoted the system divergence.

It could also be observed from Figure 2 that when $\sigma \epsilon$ $[0.02620,0.02973]$ and $k \in[7.387,14.325]$, the blue stable and the red periodic zones mingled with each other, in the range of $\sigma \in[0.02745,0.03255]$ and $k \in[-76.851,-52.573]$; the green chaotic zone was always interspersed with the red periodic zone; when $k \in[-89.895,-85.786]$, certain red periodic dots were scattered in the black diverged zone; around the interfaces between zones, the attractors were close to each other and a slight change in the system initial condition would

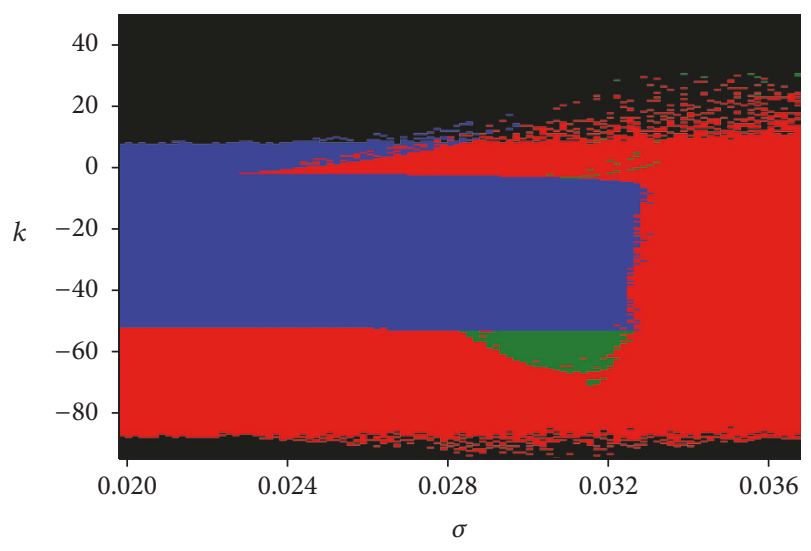

Figure 2: Dynamic map.

result in completely different dynamic behaviors; therefore the multistable phenomenon of coexisting attractors existed.

Through the dynamic map utilization, the parameter range of the vehicle stable movement could be determined. When the cavitator number $\sigma$ was constant, the stable movement of the supercavitating vehicle could be effectively achieved by the feedback control gain value adjustment of the deflection angle of fin $k$ in the corresponding range, for the vehicle stable control to be guided.

3.2. Multistable Phenomena of Supercavitating Vehicle. Based on the dynamic map in Figure 2, the generic dynamic characteristics of the supercavitating vehicle generated by the control parameters variation were discussed in our previous research [21]. In contrast, in a strongly nonlinear system such as the supercavitating vehicle, a slight disturbance on the initial condition of launching from the outside, with the system parameters retained constant, would generate the multistable phenomena.

\subsubsection{Coexistence between Stable Equilibrium Point and Limit} Cycle. Select $\sigma=0.0331, k=-19.86$ in the range of $\sigma \in[0.03226,0.03327]$ and $k \in[-50.09,-2.523]$; substitute them into (6). Equilibrium point can be obtained $Q_{1}\left(z^{*}, w^{*}\right.$, $\left.\theta^{*}, q^{*}\right)=(-0.0054,-0.1061,-0.0015,0)$. Figure 3(a) presents the projection of system on the $q-\theta-w$ plane. When the initial values were $\alpha_{1}\left(z_{0}, w_{0}, \theta_{0}, q_{0}\right)=(-0.8314,-0.9792$, $-1.1564,-0.5336$ ), the system (6) solution at the equilibrium point converged to the red stable point under the feedback control law; when the initial values were $\alpha_{2}\left(z_{0}, w_{0}, \theta_{0}, q_{0}\right)=$ 


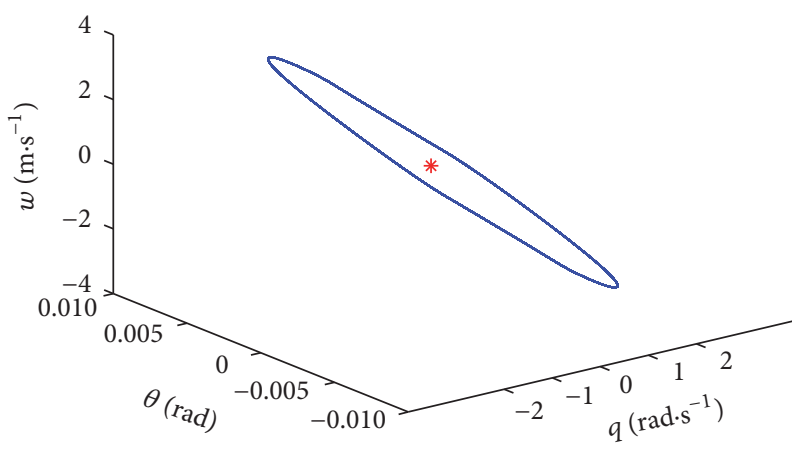

(a) Projection of coexisting attractor on $q-\theta-w$ plane

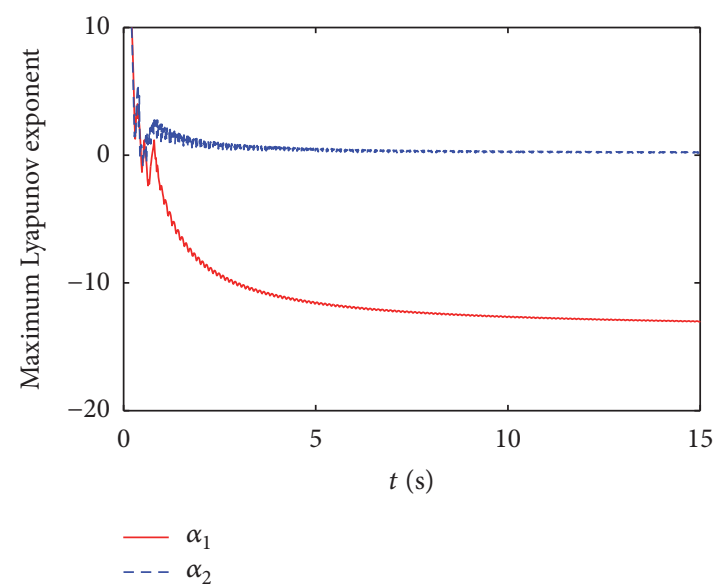

(b) Lyapunov exponent spectrum

FIGURE 3: Coexistence between stable equilibrium point and limit cycle at $\sigma=0.0331$ and $k=-19.86$.

$(-0.0229,-0.2620,-1.7502,-0.2857)$, the system (6) solution at the equilibrium point converged to the blue limit cycle attractor.

Figure 3(b) presents the Lyapunov exponent spectrum as the system evolved with time. For a clear comparison, Figure 3(b) provides the highest Lyapunov exponent curves only under two initial conditions. For initial condition $\alpha_{1}$, by numerical calculation, the finite-time local Lyapunov exponents of the system at $t=15 \mathrm{~s}$ were $L_{1}=-13.01$, $L_{2}=-13.32, L_{3}=-25.42, L_{4}=-26.09, L_{1}<L_{2}<$ $L_{3}<L_{4}<0$, whereas the maximum Lyapunov exponent $L_{1}$ was below 0 . According to the Lyapunov stability theory, the vehicle movement was stable. With the same parameters, for the initial condition $\alpha_{2}$, by numerical calculation, the finitetime local Lyapunov exponents of the system at $t=15 \mathrm{~s}$ were $L_{1}=0.08, L_{2}=-13.76, L_{3}=-16.16, L_{4}=-49.77, L_{4}<$ $L_{3}<L_{2}<0$, whereas the maximum Lyapunov exponent $L_{1}$ was approximately 0 , displaying a periodical oscillation.

Though computing the linearized Jacobi matrix at the equilibrium point of the system, the characteristic roots can be obtained as follows: $\lambda_{1,2}=-95.31 \pm j 138.90, \lambda_{3,4}=-2.72$ $+j 36.83$. Here, $\lambda_{1,2}$ and $\lambda_{3,4}$ both are conjugate imaginary roots whose real parts are negative, which indicates that the equilibrium point $Q_{1}$ is stable focus. Hidden attractor is a new class of attractor defined in recent years. It has been found in some chaotic systems that have stable equilibrium points or no equilibrium point [22-24]. Therefore, it is obvious that the periodic attractor of the supercavitating vehicle is a hidden attractor. In practical engineering, due to hidden attractor, the hidden oscillation will appear in the system, and these hidden dynamical behaviors often have an essential influence on the system's motion.

Therefore, when $\sigma=0.0331$ and $k=-19.86$, the system exhibited the coexistence among multiple attractors that were sensitive to the initial values, whereas the phase portrait tended to two types of attractors. When the system control parameters were constant, the emergence of two types of attractors demonstrated that the vehicle motion states were different under different initial conditions.
3.2.2. Coexistence between Limit Cycle and Chaotic Attractor. Select $\sigma=0.03259, k=-56.04$ in the range $\sigma \in[0.02745$, $0.03255]$ and $k \in[-76.851,-52.573]$; substitute them into (6). Equilibrium point can be obtained $Q_{2}\left(z^{*}, w^{*}, \theta^{*}, q^{*}\right)=$ $(0.0019,-0.0138,-0.0002,0)$. Figure $4($ a) presents the system projection on the $q-\theta-w$ plane. When the initial values were $\beta_{1}\left(z_{0}, w_{0}, \theta_{0}, q_{0}\right)=(-0.1924,0.8886,-0.7648,-1.4023)$, at the equilibrium point, the system (6) solution converged to a red periodic attractor; when the initial values were $\beta_{2}\left(z_{0}\right.$, $\left.w_{0}, \theta_{0}, q_{0}\right)=(-1.0616,2.3505,-0.6156,-0.7481)$, the system (6) solution converged to a blue chaotic attractor at the equilibrium point.

Figure 4(b) presents the highest Lyapunov exponent spectrum of the system evolving with time at different initial conditions. When the initial condition was $\beta_{1}$, by numerical calculation, the finite-time local Lyapunov exponents of the system at $t=15$ s were $L_{1}=0.06, L_{2}=-26.58, L_{3}=-26.76, L_{4}$ $=-27.55, L_{1} \approx 0, L_{2}<L_{3}<L_{4}<0$, whereas the maximum Lyapunov exponent $L_{1}$ was approximately 0 , demonstrating that the vehicle was in the motion state of periodic oscillation; with the same parameters, when the initial condition was $\beta_{2}$, by numerical calculation, the finite-time local Lyapunov exponents of the system at $t=15 \mathrm{~s}$ were $L_{1}=6.04, L_{2}=-0.55$, $L_{3}=-94.77, L_{4}=-97.34, L_{1}>0, L_{2}<L_{3}<L_{4}<0$, whereas the maximum Lyapunov exponent $L_{1}$ exceeded 0 , suggesting an instability of the vehicle.

Though computing the linearized Jacobi matrix at the equilibrium point of the system, the characteristic roots can be obtained as follows: $\lambda_{1,2}=-100.98 \pm j 94.67, \lambda_{3,4}=1.43$ $\pm j 76.05$. Here, $\lambda_{3,4}$ are conjugate complex roots whose real parts are positive, which indicates the equilibrium point $Q_{2}$ is unstable saddle focus. The equilibrium point $Q_{2}$ is chosen as the initial condition of the system, and the phase trajectory of the system is shown in Figure 5. A chaotic attractor appears, which corresponds to a unstable equilibrium point, indicating the chaotic attractor is a self-excited attractor. While the periodic attractors are not excited by unstable equilibrium point, they are hidden attractors. Therefore, when $\sigma=$ 0.03259 and $k=-56.04$, the phenomenon of the coexisting 


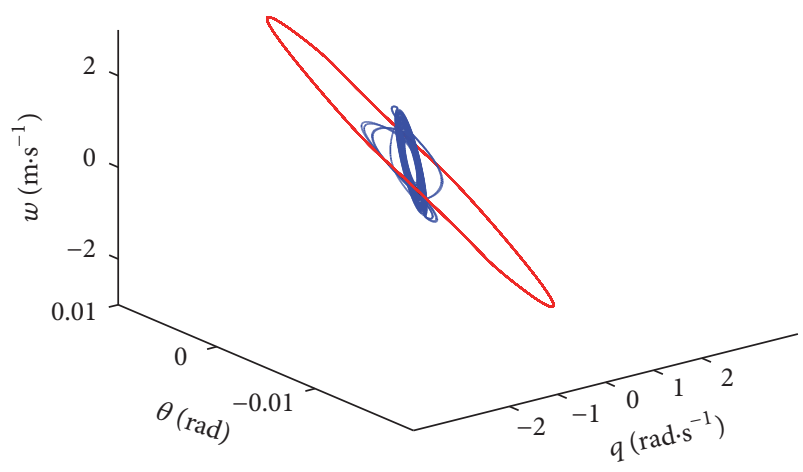

(a) Projection of coexisting attractors on $q-\theta-w$ plane

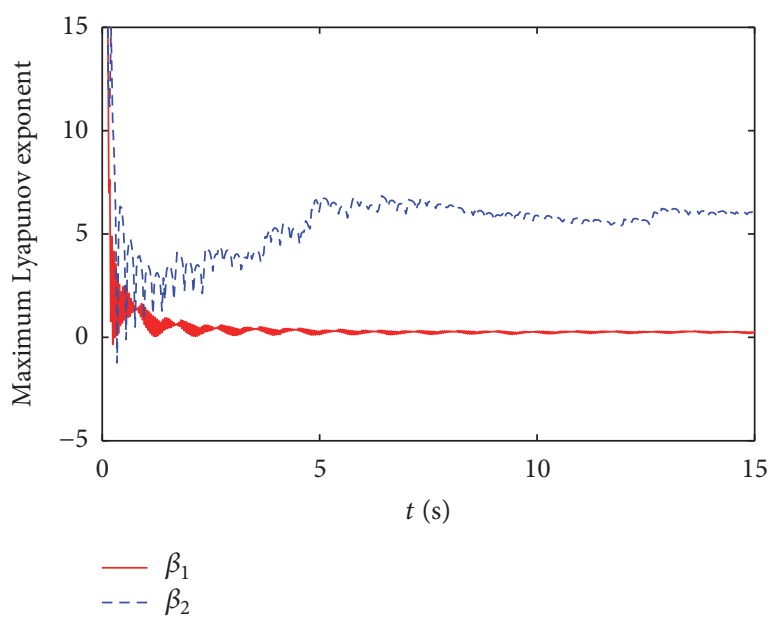

(b) Lyapunov exponent spectrum

FIgURE 4: Coexistence between limit cycle and chaotic attractors when $\sigma=0.03259$ and $k=-56.04$.

between limit cycle and chaotic attractor existed, whereas different initial conditions of the supercavitating vehicle launching would lead to different motion states of the vehicle.

\subsubsection{Coexistence among Multiple Limit Cycles. The coex-} istence of the stable equilibrium point and the limit cycle and the coexistence of the limit cycle and chaotic attractors both were common multistable phenomena in the supercavitating vehicle system. Besides, certain system parameters had other types of multistability under individual parameters, such as the coexistence among multiple limit cycles. Through calculation, when $\sigma=0.03187$ and $k=$ -70.94 , equilibrium point can be obtained $Q_{3}\left(z^{*}, w^{*}, \theta^{*}, q^{*}\right)$ $=(0.0015,-0.0275,-0.0004,0)$.

Figure 6(a) presents the system projection on the $q$ $\theta-w$ plane, when the initial condition was $\gamma_{1}\left(z_{0}, w_{0}, \theta_{0}, q_{0}\right)$ $=(0.7015,-2.0518,-0.3538,-0.8236)$. At the equilibrium point, the system (6) solution converged to the red limit cycle of period 1 . With the same parameters of the system, when the initial condition was $\gamma_{2}\left(z_{0}, w_{0}, \theta_{0}, q_{0}\right)=$ $(-0.4686,-0.2725,1.0984,-0.2779)$, the system (6) solution converged to the blue limit cycle of period 2 at the equilibrium point.

Figure 6(b) presents the maximum Lyapunov exponent spectrum of the system evolving with time at different initial conditions. When the initial condition was $\gamma_{1}$, by numerical calculation, the finite-time local Lyapunov exponents of the system at $t=15 \mathrm{~s}$ were $L_{1}=-0.03, L_{2}=-0.79, L_{3}=-56.41$, $L_{4}=-86.61, L_{1} \approx 0, L_{2}<L_{3}<L_{4}<0$, demonstrating that the vehicle was in a periodic motion state. With the same parameters, when the initial condition was $\gamma_{2}$, by numerical calculation, the finite-time local Lyapunov exponents of the system at $t=15 \mathrm{~s}$ were $L_{1}=-0.013, L_{2}=-1.02, L_{3}=-56.39$, $L_{4}=-86.84, L_{1} \approx 0, L_{2}<L_{3}<L_{4}<0$, demonstrating that the vehicle was in the other periodic motion state.

Through computing the linearized Jacobi matrix at the equilibrium point of the system, the characteristic roots can be obtained as follows, $\lambda_{1,2}=-111.16 \pm j 75.76, \lambda_{3,4}=$
$9.37 \pm j 89.4$. Here, $\lambda_{1,2}$ and $\lambda_{3,4}$ both are conjugate complex roots whose real parts are positive. Obviously, the equilibrium point $Q_{3}$ is unstable saddle focus. The equilibrium point $Q_{3}$ is chosen as the initial condition of the system, and the phase trajectory of the system is shown in Figure 7. A periodic 2-cycle attractor appears, which corresponds to a unstable equilibrium point, indicating the periodic 2 -cycle attractor is a self-excited attractor. While periodic 1-cycle attractor is hidden attractors. Therefore, when $\sigma=0.03187$ and $k=-70.94$, under different initial conditions, the vehicle oscillation period could be different and multiple limit cycles coexisted.

\section{Multistable Motion Characteristics of Supercavitating Vehicle}

To completely analyze the supercavitating vehicle system, not only the complexity of attractors, but also the complex motion characteristics of the vehicle were required to be analyzed. In previous studies, the author investigated the generic motion characteristics of the supercavitating vehicle [21]. This work was mainly focused on the multistable motion characteristics of the supercavitating vehicle through the domain of attraction, the time domain, and the frequency domain analysis.

4.1. Coexistence between Stable Equilibrium Point and Limit Cycle. According to the dynamic map in Figure 2, in the interlaced region of the blue stable zone and the red periodic zone, given that $\sigma=0.0331$, the feedback control law was $\delta_{c}$ $=15 z-30 \theta-0.3 q$ and $\delta_{e}=19.86 z$ is selected. Figure 8 presents the section of the attraction domain in $z_{0}-\theta_{0}$, which was symmetrical with regard to $\theta_{0}=z_{0}$. Through any point utilization in the domain of attraction of a certain attractor as the initial condition, the system always converged to the attractor [13]. It could be observed from Figure 8 that if the system control parameters were retained constant and the initial values of the vertical position $z_{0}$ and the pitch angle 


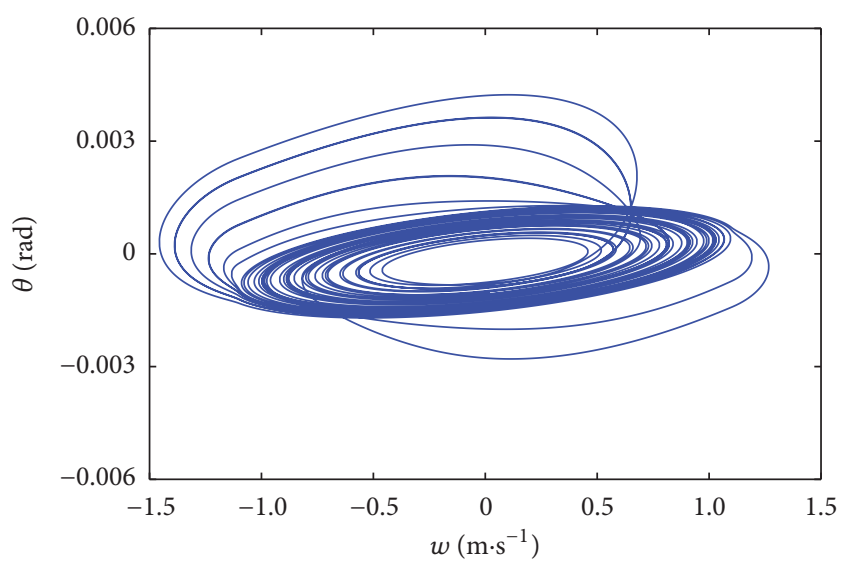

(a) $w-\theta$

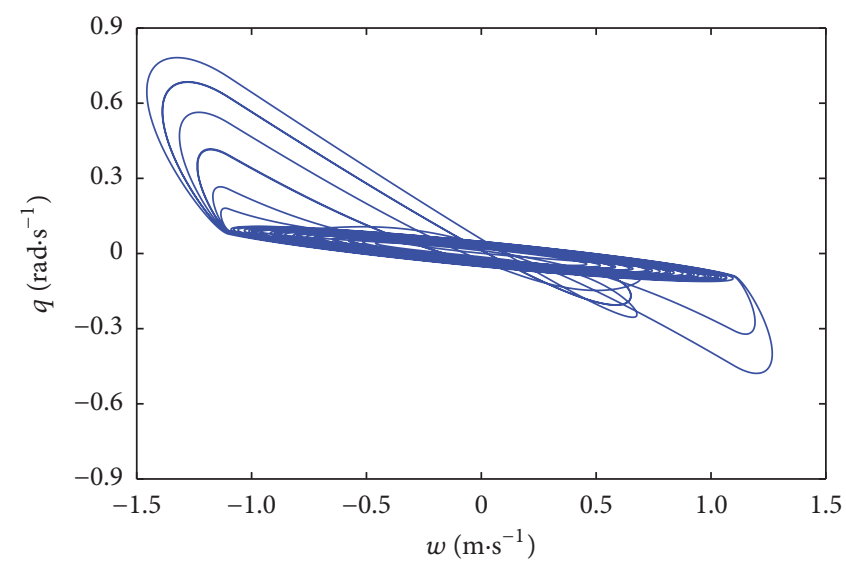

(b) $w-q$

FIGURE 5: Projections of self-excited attractor on phase plane.

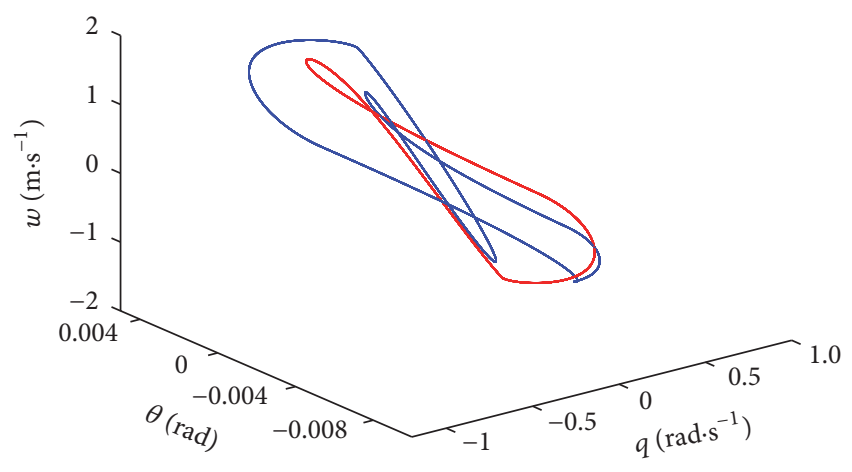

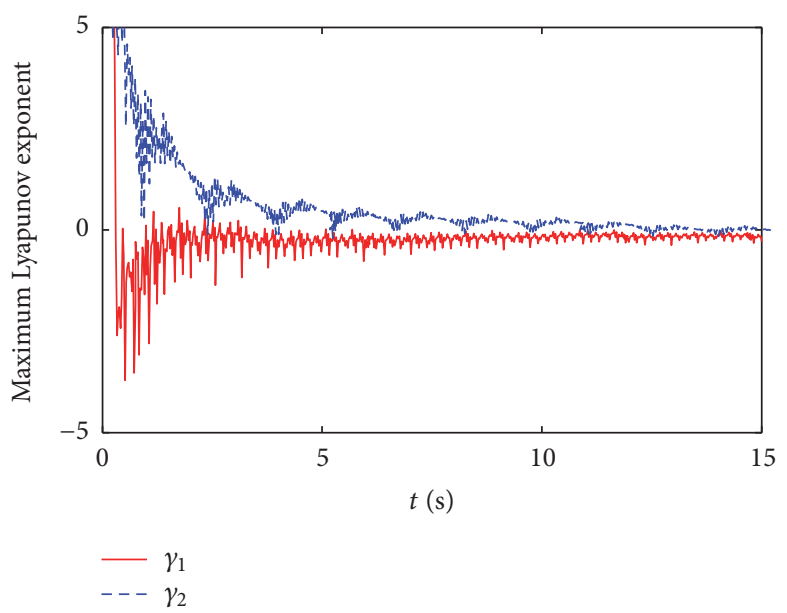

(b) Lyapunov exponent spectrum

Figure 6: Coexisting attractors of period 1 cycle and period 2 cycle when $\sigma=0.03187$ and $k=-70.94$.

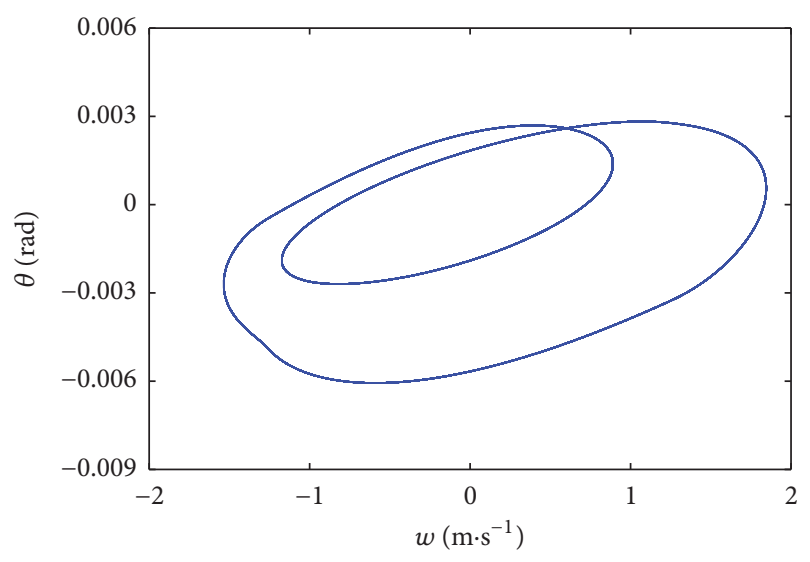

(a) $w-\theta$

FIGURE 7: Projections of self-excited attractor on phase plane. 


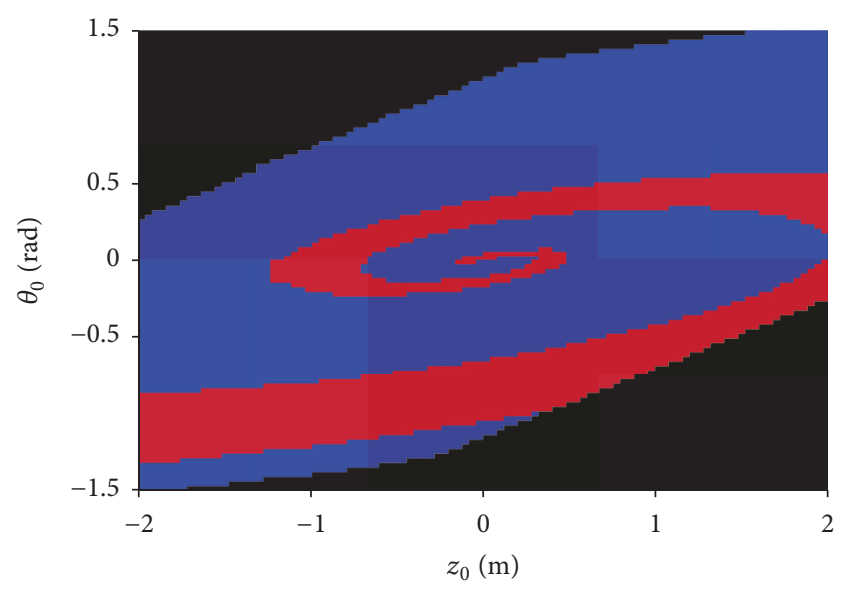

FiguRE 8: Domain of attraction for $\sigma=0.0331$ and $k=-19.86$.

$\theta_{0}$ were assigned in the blue zone, the vehicle moved stably; if the initial values corresponded to a point in the red zone, the vehicle oscillated periodically.

Consequently, it was also observed that when the initial condition was located near the boundary of the domain of attraction, a low disturbance would lead to completely different motion states of the vehicle. In practical engineering applications, the domain of attraction is of high engineering application value. Through the domain of attraction of the system, the initial condition of the supercavitating vehicle launching could be adjusted to enhance the vehicle stability.

Select the initial values $\alpha_{1}\left(z_{0}, w_{0}, \theta_{0}, q_{0}\right)=(-0.8314$, $-0.9792,-1.1564,-0.5336)$ in the blue zone and the initial values $\alpha_{2}\left(z_{0}, w_{0}, \theta_{0}, q_{0}\right)=(-0.0229,-0.2620,-1.7502$, $-0.2857)$ in the red zone in Figure 8. The simulation results of the time domains and the frequency domains of each system parameter are presented in Figure 9. The frequency domains of each variable are under the time domain figures of each variable. The real lines indicated the time domains and the frequency domains of the variations of four system state variables $z, w, \theta, q$, the immersion depth $h^{\prime}$, the planing force $F_{\text {planing }}$, the deflection angle of the cavitator $\delta_{c}$, and the deflection angle of fin $\delta_{e}$ given the initial value $\alpha_{1}$, whereas the dashed lines stood for the time domains and frequency domains of each parameter of the system given the initial value $\alpha_{2}$.

When the initial value was $\alpha_{1}$, under the control law, the state variables $z, w, \theta, q$ quickly stabilized at the equilibrium point $(-0.0054,-0.1061,-0.0015,0)$; the vehicle was located in the supercavity and the immersion depth $h^{\prime}$ of the vehicle tail penetrating the cavity and the planing force $F_{\text {planing }}$ were displayed as 0 ; the vehicle tail was inside the cavity without being in contact with the cavity. Therefore, the position and the attitude were fixed in the cavity, whereas the depth of movement was constant; consequently the vehicle was in a stable, horizontal, and straight movement state; the immersion depth $h^{\prime}$ and the planing force $F_{\text {planing }}$ corresponded to the negative infinite in the frequency spectrum, whereas the frequency spectra energy of other system parameters were located at the frequency of 0 . This demonstrated that the vehicle would be in the stable state quickly.
When the initial value was $\alpha_{2}$, the four state variables oscillated around the equilibrium point periodically; the immersion depth of the vehicle $h^{\prime}$ oscillated periodically within approximately $[0,0.08] \mathrm{m}$; in this case, under the action of gravity, the vehicle tail constantly and periodically collided with the cavity wall; sometimes the tails were in the cavity without being in contact with the cavity and sometimes the tails penetrated the water through the cavity, producing a planing force of approximately $378 \mathrm{~N}$; the $\delta_{c}$ and $\delta_{e}$ oscillated around the equilibrium point periodically; the corresponding frequency spectrum displayed several scattered peaks, demonstrating a relatively long-term significant periodic oscillation of the vehicle.

4.2. Coexistence between Limit Cycle and Chaotic Attractor. According to the dynamic map in Figure 2, within $\sigma \in$ $[0.02745,0.03255]$ and $k \in[-76.851,-52.573]$ in the interlaced region of the red periodic and the green chaotic, given that $\sigma=0.03259$, the feedback control law $\delta_{c}=15 z-30 \theta-0.3 q$ and $\delta_{e}=56.04 z$ was selected. Figure 10 presents the section of the attraction domain in the $z_{0}-\theta_{0}$, whereas the fragment boundaries were in the domain of attraction. The red zone represented the initial values that would lead to the final arrival on the periodic orbit and the periodic oscillation of the vehicle; the green zone stood for the initial values that would finally fall into chaos. It could be observed from Figure 10 that a slight change in the initial condition of launching led to a motion transition of the vehicle from a stable periodic motion state to a nonperiodic oscillation state. In certain practical applications, the supercavitating vehicle motion in these variable parameter combinations should be avoided.

Select the initial values $\beta_{1}\left(z_{0}, w_{0}, \theta_{0}, q_{0}\right)=(-0.1924$, $0.8886,-0.7648,-1.4023)$ in the red zone and the initial values $\beta_{2}\left(z_{0}, w_{0}, \theta_{0}, q_{0}\right)=(-1.0616,2.3505,-0.6156,-0.7481)$ in the green zone in Figure 10; the simulation results of the motion characteristics of the vehicle are presented in Figure 11. In this case, the real lines represented the time domains and the frequency domains of the variations of four system state variables $z, w, \theta, q$, the immersion depth $h^{\prime}$, the planing force $F_{\text {planing }}$, the deflection angle of the cavitator $\delta_{c}$, and the deflection angle of fin $\delta_{e}$ given initial value $\beta_{1}$, whereas the dashed lines represented the time domains and the frequency domains of each parameter of the system given the initial value $\beta_{2}$.

As presented in Figure 11, when the initial value was $\beta_{1}$, the four system state variables $z, w, \theta$, and $q$ oscillated around the equilibrium point $(0.0019,-0.0138,-0.0002,0)$ periodically. Under the action of gravity, the tail of the vehicle collided with the cavity wall and penetrated the water, for the planing force to be produced; as the immersion depth increased, the tail of the vehicle was quickly bounced back into the cavity under planing force and collided with the other side of the cavity wall. Also, the cycle was repeated; $\delta_{c}$ and $\delta_{e}$ also oscillated around the equilibrium point periodically; the corresponding frequency spectra were all scattered sharp peaks with equal intervals, whereas all these demonstrated that the vehicle periodically oscillated in the cavity. Nevertheless, a periodic collision between the tail and the cavity wall caused the strong nonlinear planing force. The 

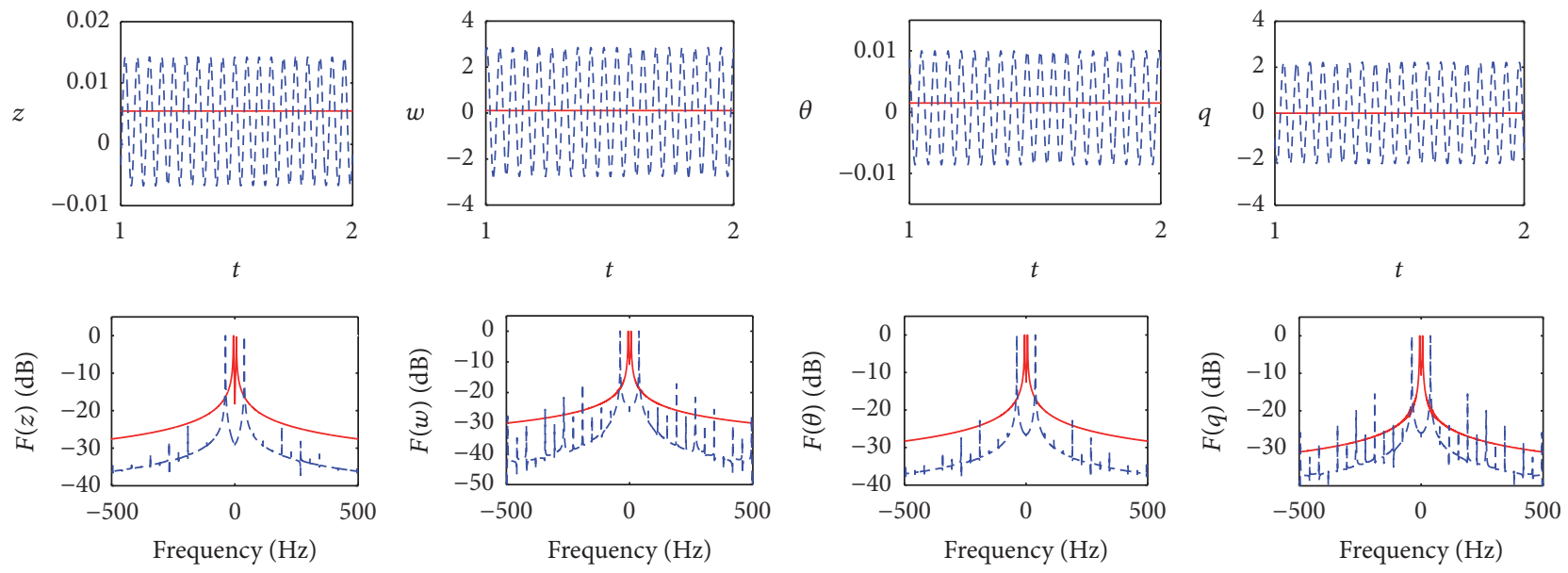

(a) Time domain and frequency domain graphs of $z, w, \theta, q$
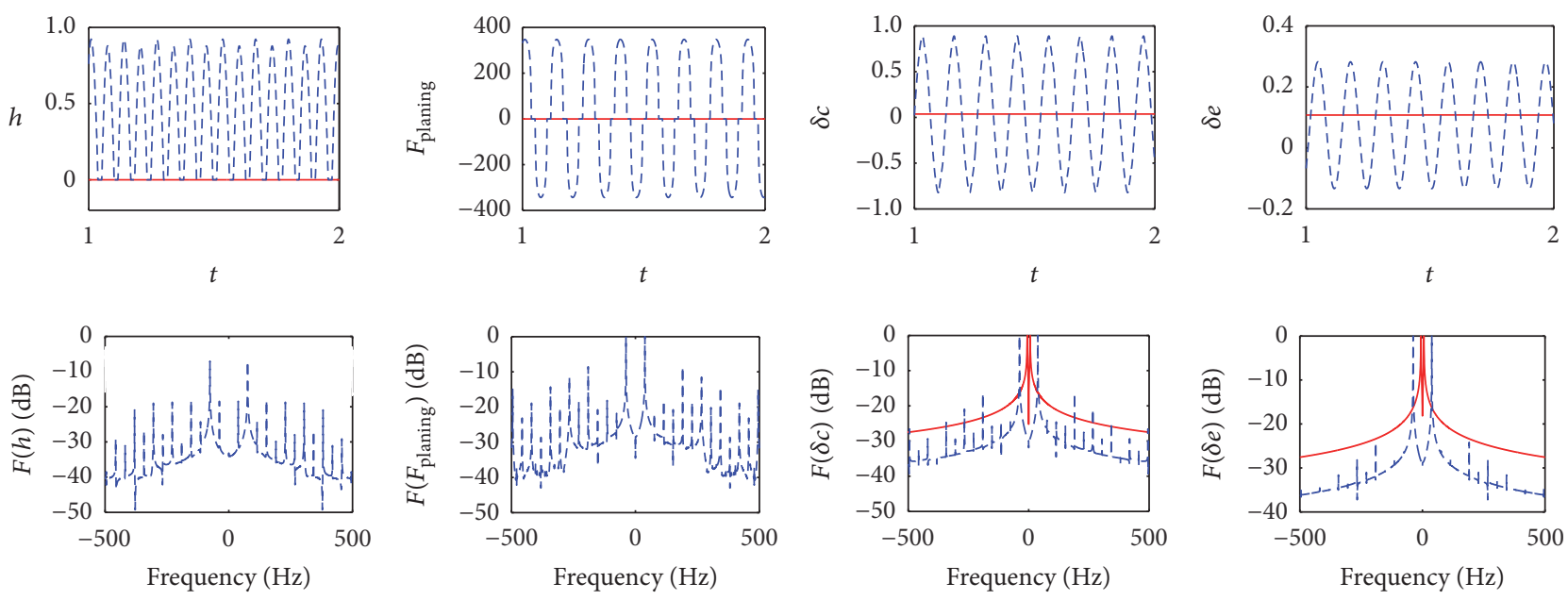

(b) Time domain and frequency domain graphs of $h^{\prime}, F_{\text {planing }}, \delta_{c}, \delta_{e}$

Figure 9: Time domain and frequency domain graphs at $\sigma=0.0331$ and $k=-19.86$.

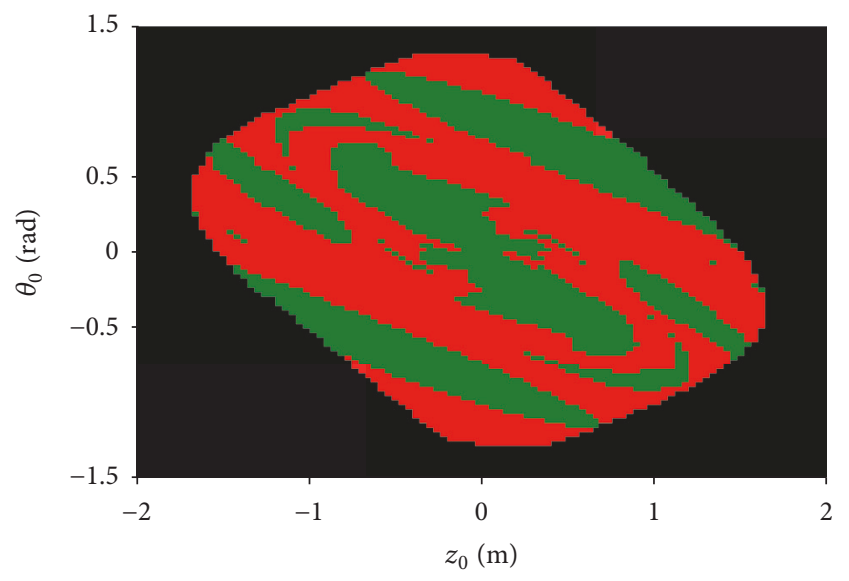

FIGURE 10: Domain of attraction for $\sigma=0.03259$ and $k=-56.04$.

planing force existence would cause dramatic vibration and shock to the vehicle, consequently leading to a loss of stability and furthermore the vehicle overturning.
When the initial value was $\beta_{2}$, the state variables of the vehicle oscillated nonperiodically around the equilibrium point as the duration varied; the planing force $F_{\text {planing }}$ and the immersion depth $h^{\prime}$ slightly nonperiodically oscillated, demonstrating that the tail of the vehicle slightly collided with the cavity wall; however, $\delta_{c}$ and $\delta_{e}$ exhibited significant nonperiodic vibration; also, the frequency domains corresponding to the system parameters all displayed wideband continuous spectra, demonstrating that the vehicle in the cavity was in a nonperiodic oscillation state. Since each system parameter was in the nonperiodic unstable state, the vehicle motion was also vulnerable to external disturbance and thereby led to loss of stability.

4.3. Coexistence among Multiple Limit Cycles. When $\sigma=$ 0.03187 and the feedback control law $\delta_{c}=15 z-30 \theta-0.3 q, \delta_{e}$ $=70.94 z$ was selected, the cross section of the domain of attraction on the $z_{0}-\theta_{0}$ is presented in Figure 12, where the red zone represented the initial values resulting in the period 1 orbit and the yellow zone for the initial values resulting 

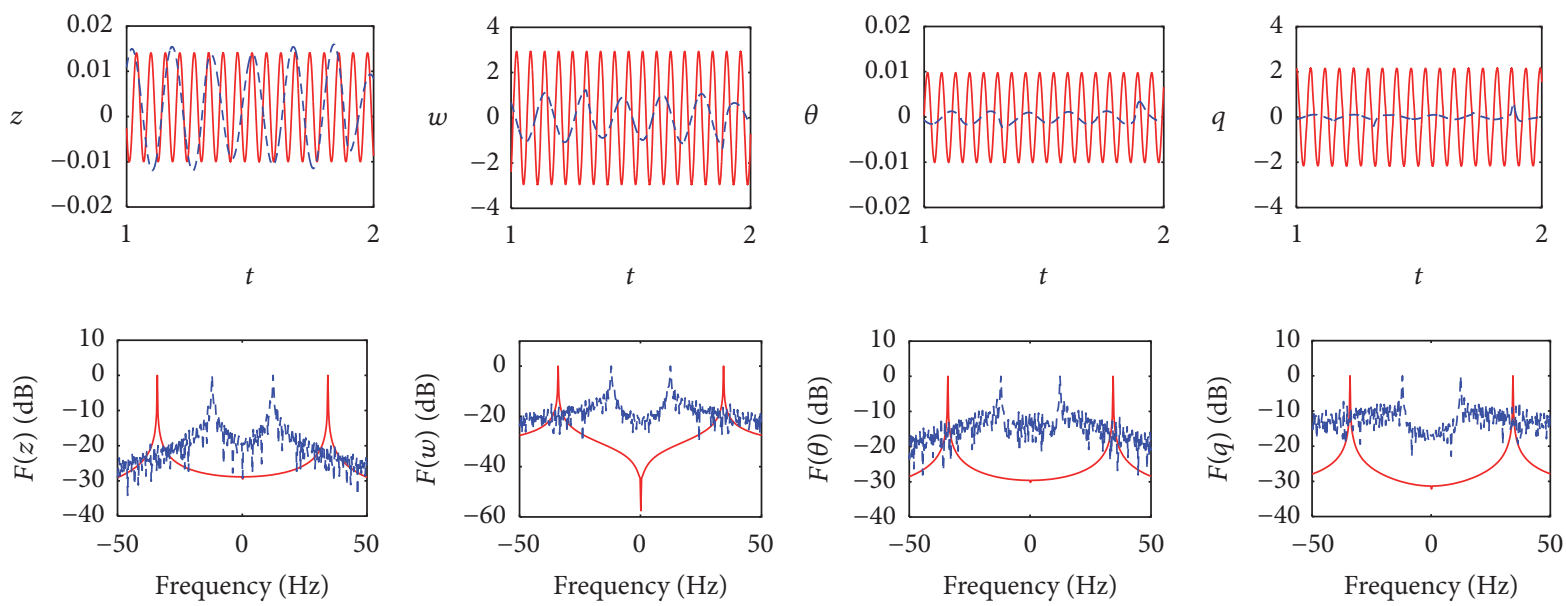

(a) Graphs of time domain and frequency domain of $z, w, \theta, q$
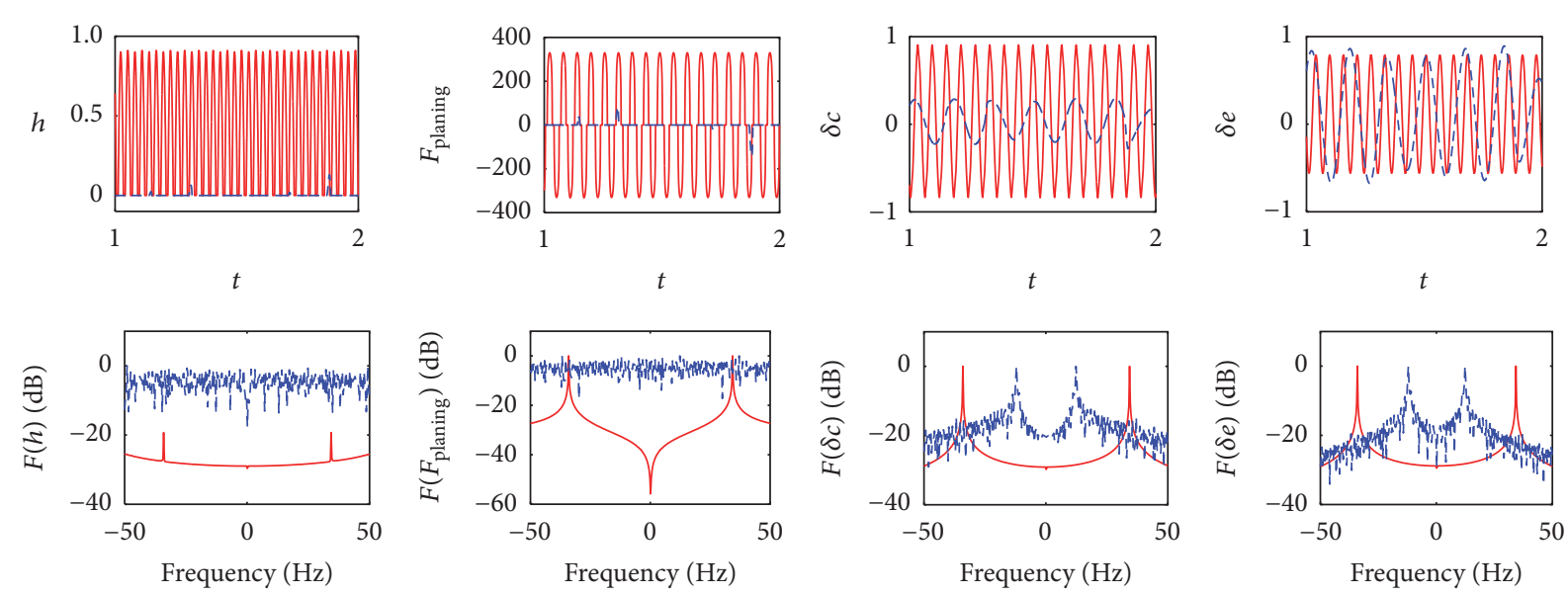

(b) Graphs of time domain and frequency domain of $h^{\prime}, F_{\text {planing }}, \delta_{c}, \delta_{e}$

FIGURE 11: Graphs of time domain and frequency domain at $\sigma=0.03259$ and $k=-56.04$.

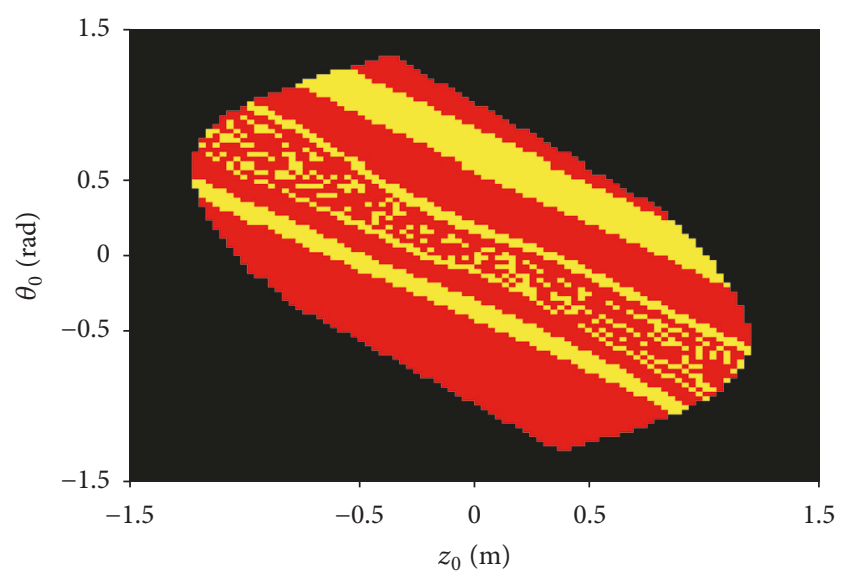

FIGURE 12: Domain of attraction at $\sigma=0.03187$ and $k=-70.94$.

in period 2 orbit. As the initial condition varied, the vehicle displayed different periodic oscillations.
Select the initial values $\gamma_{1}\left(z_{0}, w_{0}, \theta_{0}, q_{0}\right)=(0.7015$, $-2.0518,-0.3538,-0.8236)$ in the red zone and $\gamma_{2}\left(z_{0}, w_{0}\right.$, $\left.\theta_{0}, q_{0}\right)=(-0.4686,-0.2725,1.0984,-0.2779)$ in the yellow zone in Figure 12; the simulation results of the vehicle motion characteristics are presented in Figure 13. In this case, the real lines indicated the time domains and the frequency domains of the variations of four system state variables $z, w$, $\theta, q$, the immersion depth $h^{\prime}$, the planing force $F_{\text {planing }}$, the deflection angle of the cavitator $\delta_{c}$, and the deflection angle of fin $\delta_{e}$ given the initial value $\gamma_{1}$, whereas the dashed lines represented the time domains and the frequency domains of each parameter of the system given the initial value $\gamma_{2}$.

As presented in Figure 13, for all initial values, the four system state variables $z, w, \theta$, and $q$ oscillated around the equilibrium point $(0.0015,-0.0275,-0.0004,0)$ periodically. When the initial value was $\gamma_{1}$, the vehicle tail periodically collided with the lower wall of the cavity and the immersion depth of the vehicle tail $h^{\prime}$ oscillated around $0.25 \mathrm{~m}$. Therefore, the resulting planing force $F_{\text {planing }}$ also oscillated around $200 \mathrm{~N}$; when the initial value was $\gamma_{2}$, the collision between the vehicle tail and both the upper and lower 

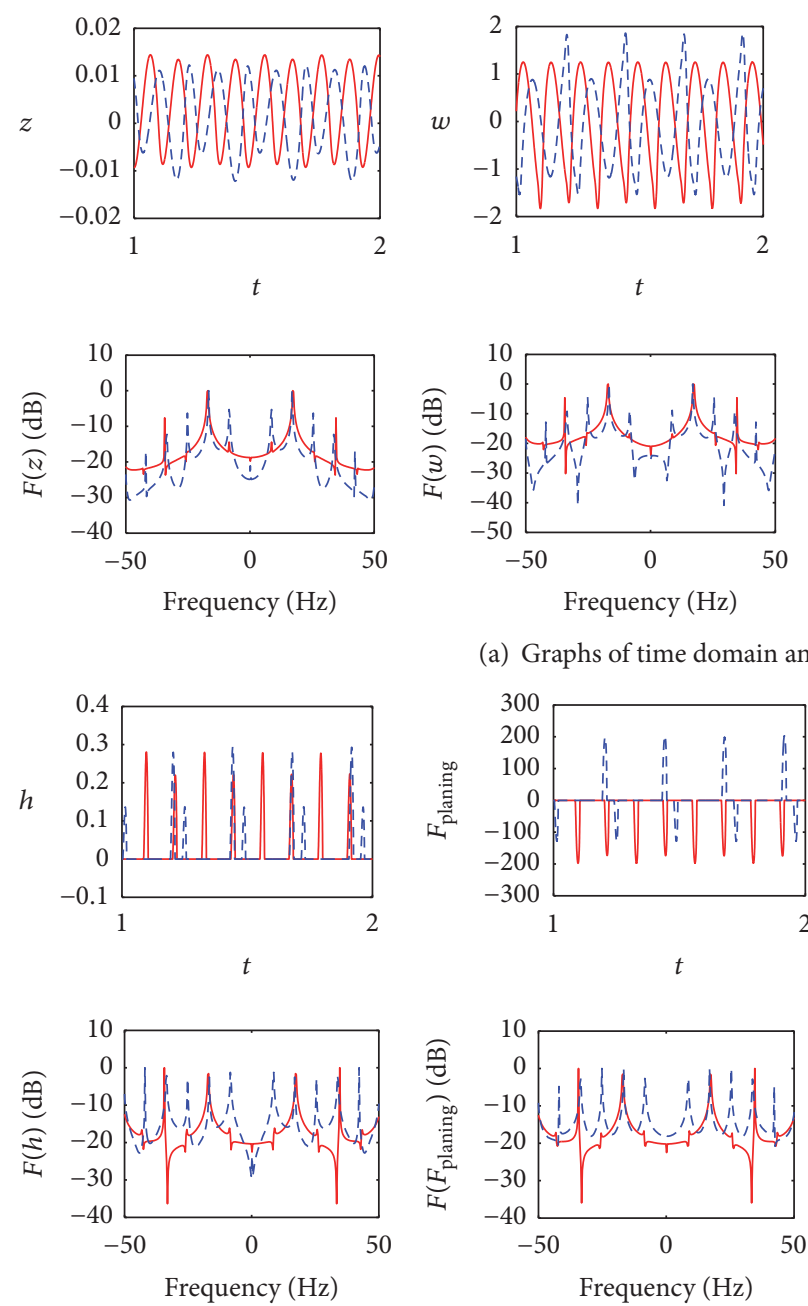
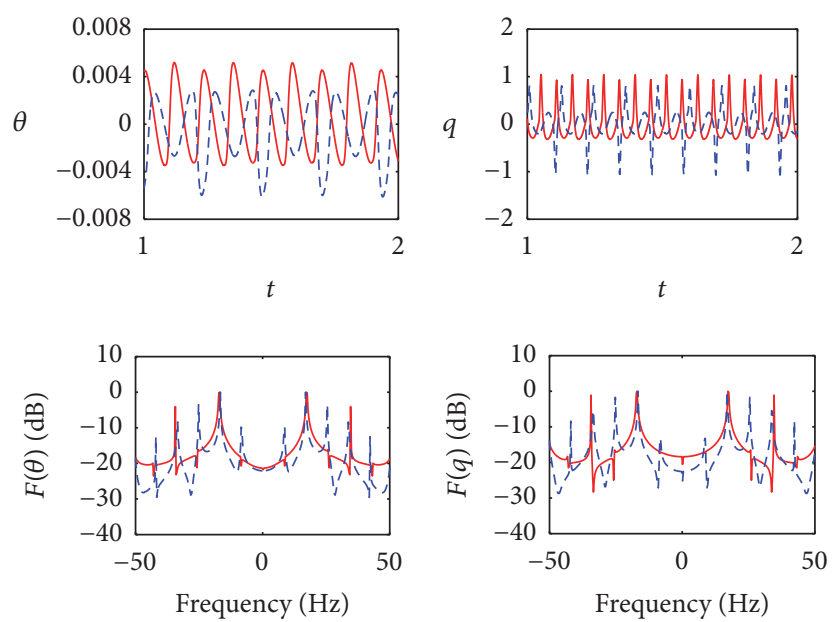

(a) Graphs of time domain and frequency domain of $z, w, \theta, q$
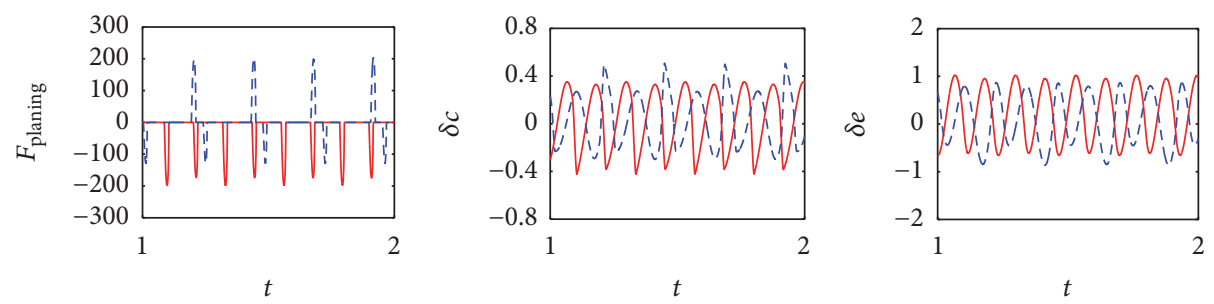

(b) Graphs of time domain and frequency domain of $h^{\prime}, F_{\text {planing }}, \delta_{c}, \delta_{e}$
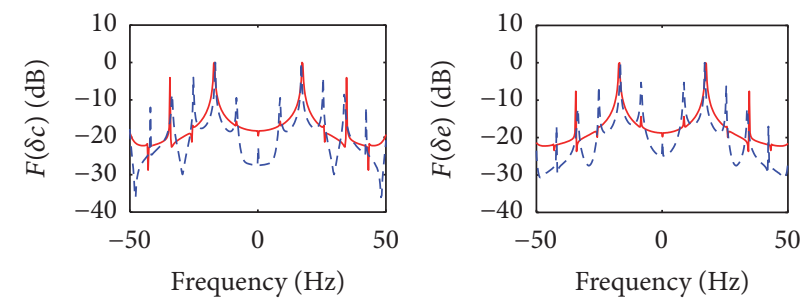

FIGURE 13: Graphs of time domain and frequency domain at $\sigma=0.03187$ and $k=-70.94$.

walls of the cavity was asymmetric periodic. The periodic variation of the immersion depth $h^{\prime}$ in $[0.12-0.3] \mathrm{m}$ led to $F_{\text {planing }}$ in the [-132-201] N and thereby the vehicle tail periodically oscillated outside the cavity; different initial conditions all corresponded to the frequency spectra of the scattered peaks of equal intervals. Consequently, two different types of periodic oscillation of the vehicle in the cavity were observed.

From the aforementioned analyses, it could be concluded that the motion state of the supercavitating vehicle depended extremely on the system initial condition. Different initial conditions of launching led to the multistable motion characteristics. In practical engineering applications, due to the complex underwater environment, the external disturbance factors are diversified [25]. Under fixed system parameters, as the initial condition of launching was affected by slight external disturbance, the motion trace tended to display different motion states, which would be of practical engineering significance to the stable control of multistable phenomena of the vehicle.

\section{Conclusions}

Based on dynamic map of the supercavitating vehicle, the multistable phenomena of the attractor coexistence of the supercavitating vehicle were analyzed, whereas the following conclusions were drawn through the multistable motion characteristics discussion of the vehicle with the domain of attraction, the time domain, and the frequency domain:

(1) Unlike ordinary dynamic systems, the supercavitating vehicle system has multistable phenomena, such as the coexistence between the stable equilibrium point and the limit cycle, between the limit cycle and the chaotic attractor, and among multiple limit cycles.

(2) The motion state of the supercavitating vehicle depended extremely on the initial condition. Under fixed system parameters, as the initial condition of launching varied, the motion states of the vehicle were different.

(3) Through the domain of attraction utilization of the system, the relationship between the initial condition and the vehicle motion characteristics could be determined; in 
practical engineering applications, the motion stability of the vehicle could be enhanced through the initial launching condition adjustment according to the domain of attraction.

\section{Conflicts of Interest}

The authors declare that there are no conflicts of interest regarding the publication of this paper.

\section{Acknowledgments}

This work is supported by the National Natural Science Foundation of China (Grant nos. 11402116 and 11472163) and the Fundamental Research Funds for the Central Universities (Grant no. 30910612203).

\section{References}

[1] A. Kubota, H. Kato, and H. Yamaguchi, "A new modelling of cavitating flows: A numerical study of unsteady cavitation on a hydrofoil section," Journal of Fluid Mechanics, vol. 240, no. 3, pp. 59-96, 1992.

[2] Y. N. Savchenko, "Modeling the supercavitation processes," International Journal of Fluid Mechanics Research, vol. 28, no. 5, pp. 644-659, 2001.

[3] J. Gu, Z. Zhang, Y. Gao, and X. Zheng, "Experimental study about the influence of ventilated headforms on the drag characters of a super cavitation axisymmetric body," Binggong Xuebao/Acta Armamentarii, vol. 25, no. 6, pp. 766-769, 2004.

[4] A. K. Singhal, M. M. Athavale, H. Li, and Y. Jiang, "Mathematical basis and validation of the full cavitation model," Journal of Fluids Engineering, vol. 124, no. 3, pp. 617-624, 2002.

[5] A. Ducoin, B. Huang, and Y. L. Young, "Numerical modeling of unsteady cavitating flows around a stationary hydrofoil," International Journal of Rotating Machinery, vol. 2012, Article ID 215678, 17 pages, 2012.

[6] Q. T. Li, Y. S. He, and L. P. Xue, "A numerical simulation of pitching motion of the ventilated supercaviting vehicle around its nose," Chinese Journal of Hydrodrnamics, vol. 26, no. 6, pp. 589-685, 2011.

[7] J. Dzielski and A. Kurdila, "A benchmark control problem for supercavitating vehicles and an initial investigation of solutions," Journal of Vibration \& Control, vol. 9, no. 7, pp. 791804, 2003.

[8] G. Lin, B. Balachandran, and E. H. Abed, "Nonlinear dynamics and bifurcations of a supercavitating vehicle," IEEE Journal of Oceanic Engineering, vol. 32, no. 4, pp. 753-761, 2007.

[9] V. Nguyen and B. Balachandran, "Supercavitating vehicles with noncylindrical, nonsymmetric cavities: Dynamics and instabilities," Journal of Computational and Nonlinear Dynamics, vol. 6, no. 4, Article ID 041001, 2011.

[10] Y. T. Han, B. C. Qiang, Y. Sun, and T. Bai, "Nonlinear control ressearch on supercavitating vehicles based on circle criterion," Engineering Mechanics, vol. 32, no. 8, pp. 236-242, 2015.

[11] T. Bai, Z. Wu, Y. H. Jiang, and Y. T. Han, "Control of longitudinal motion for supercavitating vehicle based on water tunnel experiment," 2017, http://kns.cnki.net/kcms/detail/23.1390.U .20170526.1838.006.html.

[12] C. Li, J. C. Sprott, and Y. Mei, "An infinite 2-D lattice of strange attractors," Nonlinear Dynamics, vol. 89, no. 4, pp. 2629-2639, 2017.
[13] C. Li, J. C. Sprott, W. Hu, and Y. Xu, "Infinite Multistability in a Self-Reproducing Chaotic SYStem," International Journal of Bifurcation and Chaos, vol. 27, no. 10, Article ID 1750160, 2017.

[14] G. A. Leonov, N. V. Kuznetsov, and T. N. Mokaev, "Homoclinic orbits, and self-excited and hidden attractors in a Lorenzlike system describing convective fluid motion," The European Physical Journal Special Topics, vol. 224, no. 8, pp. 1421-1458, 2015.

[15] B. Bao, F. Hu, M. Chen, Q. Xu, and Y. Yu, "Self-excited and hidden attractors found simultaneously in a modified chua's circuit," International Journal of Bifurcation and Chaos, vol. 25, no. 5, Article ID 1550075, 2015.

[16] C. Li, X. Wang, and G. Chen, "Diagnosing multistability by offset boosting," Nonlinear Dynamics, vol. 90, no. 2, pp. 13351341, 2017.

[17] J. C. A. de Bruin, A. Doris, N. van de Wouw, W. P. M. H. Heemels, and H. Nijmeijer, "Control of mechanical motion systems with non-collocation of actuation and friction: A Popov criterion approach for input-to-state stability and set-valued nonlinearities," Automatica, vol. 45, no. 2, pp. 405-415, 2009.

[18] M. A. Kiseleva, N. V. Kuznetsov, G. A. Leonov, and P. Neittaanmäki, "Drilling systems failures and hidden oscillations," in Proceedings of the IEEE 4th International Conference on Nonlinear Science and Complexity, NSC 2012, pp. 109-111, hun, August 2012.

[19] G. A. Leonov, N. V. Kuznetsov, O. A. Kuznetsova, S. M. Seledzhi, and V. I. Vagaitsev, "Hidden oscillations in dynamical systems," WSEAS Transactions on Systems and Control, vol. 6, no. 2, pp. 54-67, 2011.

[20] T. Lauvdal, R. M. Murray, and T. I. Fossen, "Stabilization of integrator chains in the presence of magnitude and rate saturations; a gain scheduling approach," in Proceedings of the 36th IEEE Conference on Decision and Control, pp. 4004-4005, San Diego, Calif, USA, 1997.

[21] T. Xiong, X. Li, Y. Lv, and W. Yi, "Research on the numerical simulation of the nonlinear dynamics of a supercavitating vehicle," Shock and Vibration, vol. 2016, Article ID 8268071, 2016.

[22] C. Li and J. C. Sprott, "Coexisting hidden attractors in a 4-D simplified lorenz system," International Journal of Bifurcation and Chaos, vol. 24, no. 3, Article ID 1450034, 2014.

[23] G. A. Leonov, N. V. Kuznetsov, and T. N. Mokaev, "Hidden attractor and homoclinic orbit in Lorenz-like system describing convective fluid motion in rotating cavity," Communications in Nonlinear Science and Numerical Simulation, vol. 28, no. 1-3, pp. 166-174, 2015.

[24] H. Bao, B. C. Bao, Y. Lin, J. Wang, and H. G. Wu, "Hidden attractor and its dynamical characteristic in memristive selfoscillating system," Acta Physica Sinica, vol. 65, no. 18, Article ID 180501, 2016.

[25] W. J. Yi, M. Zhang, Z. H. Chen, and J. J. Tan, "Investigations on the velocity measurement of undewater high-speed vehicle," in Proceedings of the 9th National Congress on Hydrodynamics and 22nd National Conference on Hydrodynamics, pp. 353-358, Chendu, Sichuan, China, August 2009. 


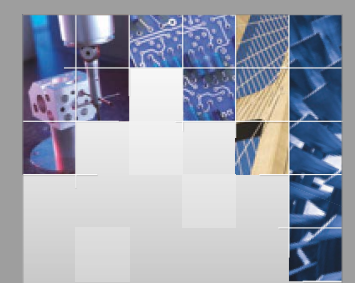

\section{Enfincering}
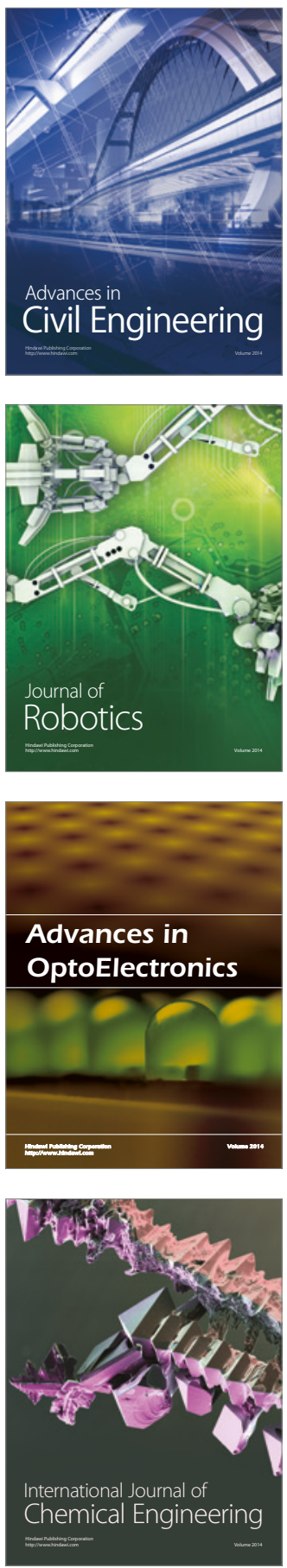

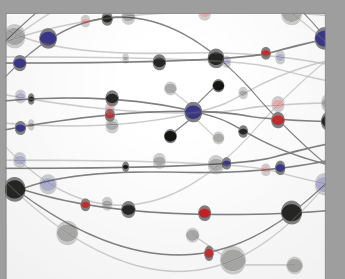

The Scientific World Journal

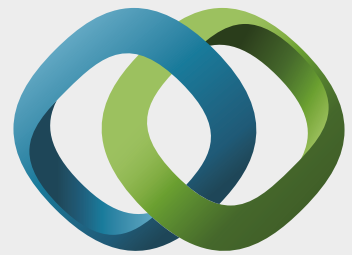

\section{Hindawi}

Submit your manuscripts at

https://www.hindawi.com
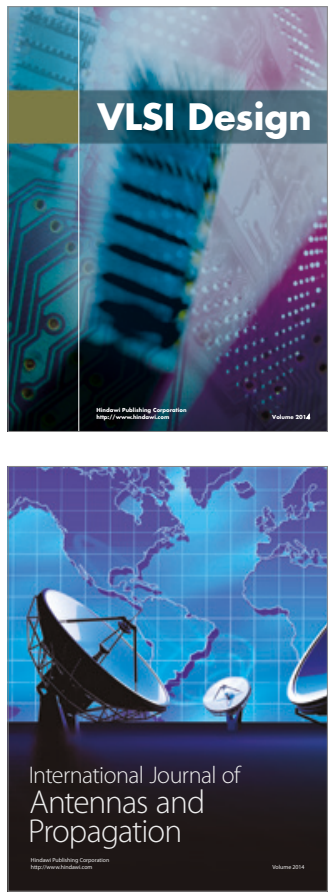

\section{Rotating}

Machinery
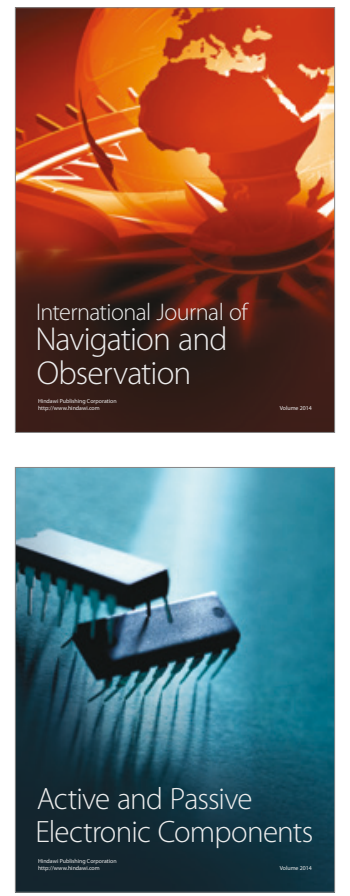
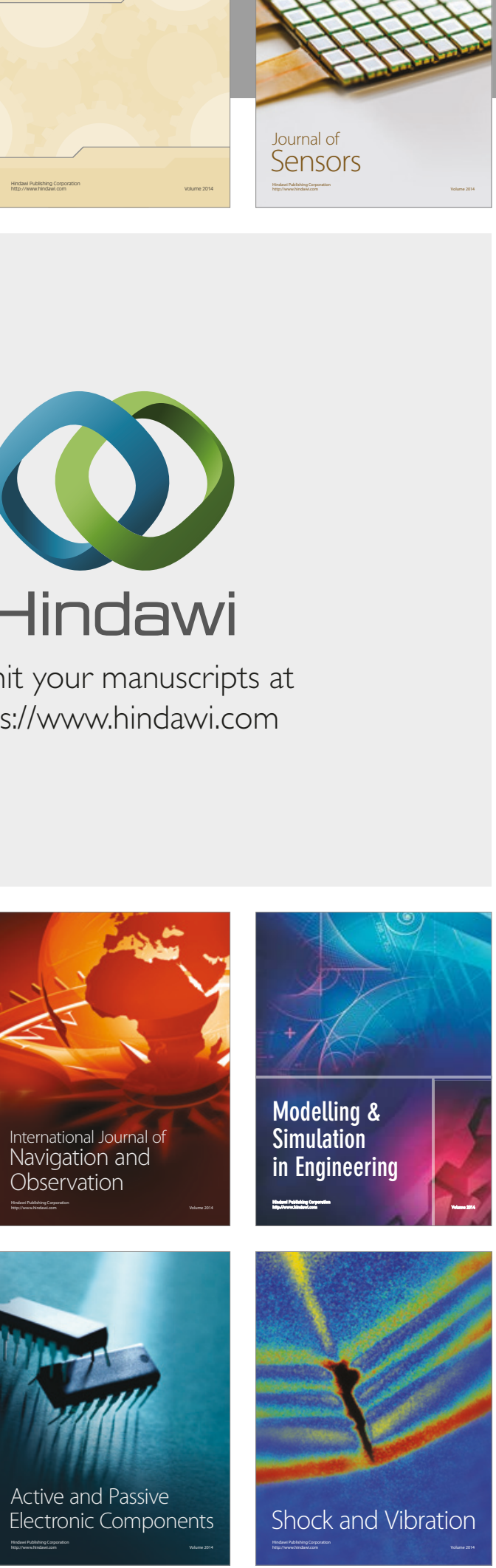
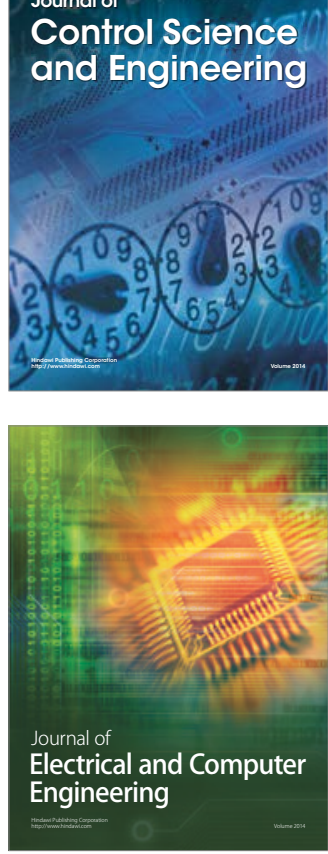

Distributed

Journal of

Control Science

and Engineering
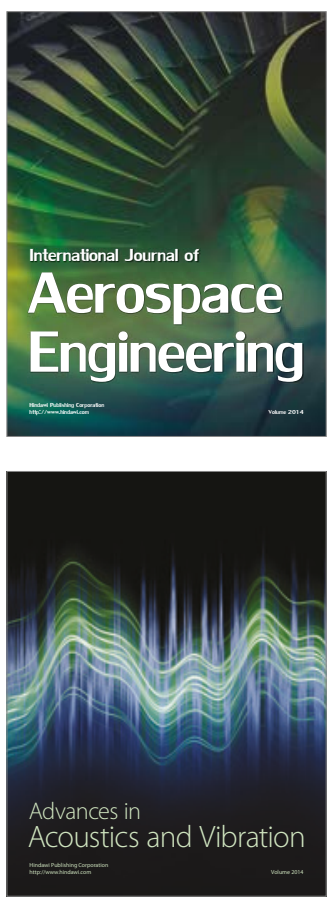

Sensor Networks 